\title{
FRACTIONAL GABOR SPECTRAL ESTIMATION FOR MULTI-COMPONENT SIGNALS
}

\author{
Erol Önen and Aydin Akan \\ Department of Electrical and Electronics Engineering, Istanbul University \\ Avcilar 34850, Istanbul, TURKEY \\ phone: +90 212473 7070/17914, fax. +90 212591 1997, e-mail: \{eerol,akan\}@istanbul.edu.tr. \\ web: www.istanbul.edu.tr/eng/ee/aydin/
}

\begin{abstract}
We present a high resolution time-frequency spectral analysis method for multi-component signals based on a discrete fractional Gabor expansion. The proposed expansion uses the closed form discrete fractional Fourier kernel and generate a parallelogram-shaped time-frequency plane tiling. Completeness and biorthogonality conditions of the new expansion are derived. We also present a search algorithm to obtain optimal analysis fraction orders for an arbitrary, multicomponent signal to estimate a high resolution Gabor spectrum.
\end{abstract}

\section{INTRODUCTION}

Time-frequency (TF) analysis provides a distribution of signal energy over the joint time-frequency plane $[2,1]$. One of the fundamental issues in the TF analysis is obtaining the distribution of signal energy over joint TF plane with sufficient time and frequency resolution (ideally with delta function concentration.) One could use impulses in the TF plane to represent a signal, however they are not feasible. Gabor proposed to use time and frequency shifted Gauss windows as basis functions because of their optimal TF concentration. Hence the Gabor expansion represents a signal as a combination of time and frequency translated basis functions, called TF atoms citeWexler. This type of basis functions generate a fixed and rectangular TF plane sampling. However, if the signal to be represented is not modeled well by this constantbandwidth analysis, its Gabor representation displays poor TF localization $[4,5,6]$. Many of the real-world signals such as speech, music, machine vibrations, biological, and seismic signals however, have time-varying frequency content that is not appropriate for sinusoidal analysis $[5,6,7]$. Thus the Gabor expansion of such signals will require large number of Gabor coefficients yielding a poor TF localization. The compactness of the Gabor representation is improved if the basis functions match the time-varying frequency behavior of the signal $[8,9]$. Several approaches have been proposed to improve the resolution of Gabor representations: some of them are using large dictionary of basis functions [5, 8], averaging results obtained using different windows [7], maximizing energy concentration measures [4], and using signaladaptive basis functions to match the time-varying frequency of the signal [9]. In recent works, representations on a nonrectangular TF grid have attracted a considerable attention [10]. A non-rectangular lattice is more appropriate for the TF analysis of signals with time-varying frequency content.

This work was supported by The Research Fund of The University of Istanbul, Project numbers: UDP-145/23052003 and 107/15052003.
Here we present a discrete fractional Gabor expansion on such a sampling scheme. The basis functions of the proposed expansion are obtained from the closed-form discrete fractional Fourier transform [11] kernel.

\section{BACKGROUND}

In this section we give brief background on the discrete Gabor expansion and fractional Fourier analysis.

\subsection{Discrete Gabor Expansion}

The traditional Gabor expansion [3] represents a signal in terms of time and frequency shifted basis functions, and has been used in various applications to analyze the time-varying frequency content of a signal [7]. The discrete Gabor expansion of a finite-support signal $x(n), 0 \leq n \leq N-1$ is given by

$$
x(n)=\sum_{m=0}^{M-1} \sum_{k=0}^{K-1} a_{m, k} \tilde{h}_{m, k}(n)
$$

where the basis functions are

$$
\tilde{h}_{m, k}(n)=\tilde{h}(n-m L) e^{j \omega_{k} n}
$$

and $\omega_{k}=2 \pi k L^{\prime} / N$. The synthesis window $\tilde{h}(n)$ is a periodic extension (by N) of $h(n)$ which is normalized to unit energy for definiteness. Gabor sampling parameters $M, K, L$, and $L^{\prime}$ are positive integers constrained by $M L=K L^{\prime}=N$ where $M$ and $K$ are the number of analysis samples in time and frequency, respectively, and $L$ and $L^{\prime}$ are the time and frequency steps, respectively. For numerically stable representations, $L$ and $L^{\prime}$ must satisfy $L L^{\prime} \leq N$, or equivalently that $L \leq K$. The case where $L=K$, is called the critical sampling, and the case $L<K$ is called the over-sampling. In general, Gabor windows $\left\{\tilde{h}_{m, k}(n)\right\}$ constitutes a non-orthogonal basis for the space of square summable sequences $\ell_{2}(N)$. Hence, the Gabor coefficients can be evaluated by using an auxiliary function called the bi-orthogonal window or analysis function [3]:

$$
a_{m, k}=\sum_{n=0}^{N-1} x(n) \tilde{\gamma}_{m, k}^{*}(n)
$$

where the basis functions are $\tilde{\gamma}_{m, k}(n)=\tilde{\gamma}(n-m L) e^{j \omega_{k} n}$ and $\tilde{\gamma}(n)$ is again a periodic version of the analysis window $\gamma(n)$ that is solved from a discrete biorthogonality condition between the analysis and synthesis bases sets [3].

Above Gabor basis $\left\{\tilde{\gamma}_{m, k}(n)\right\}$ with a fixed window and sinusoidal modulation generates a uniform and rectangular TF 
sampling lattice. Here we propose a discrete fractional Gabor expansion that uses a non-rectangular TF sampling grid, using the closed-form discrete fractional Fourier transform kernel [11]. A general, non-rectangular TF lattice is more appropriate for the analysis of signals with time-varying frequency content.

In [12], authors presented a continuous-time fractional Gabor expansion using basis functions similar to the kernel of the fractional Fourier transform (FRFT). FRFT provides a rotation of the TF plane by an angle $\alpha$, and can be used to generate basis functions with linear instantaneous frequencies [12]. Quite amount of efforts have been made to define a discrete version of the FRFT [11]. Here, we choose and employ the closed-form discrete FRFT that carries out all the desired properties of the continuous FRFT kernel.

\subsection{Discrete Fractional Fourier Transform}

In [11], a closed-form discrete FRFT is given and it was shown that the kernel of this transform is orthogonal, unitary, and invertible. Basically, it is given by sampling the original kernel of the FRFT. However, the sampling is done in such a way that the signal to be transformed and the final transform are sampled using the same sampling parameters. Hence the transformation matrix obtained in this way is orthogonal. The input signal $f(t)$ and the transform $F_{\alpha}(u)$ are sampled by sampling intervals $\Delta t$ and $\Delta u n=-N,-N+1, \cdots, N-1, N$ and $m=-M, M+1, \cdots, M-1, M$ as,

$$
y(n)=f(n \Delta t), \quad Y_{\alpha}(m)=F_{\alpha}(m \Delta u)
$$

Thus, the discrete FRFT of $y(n)$ is defined as [11],

$$
\begin{aligned}
Y_{\alpha}(m) & =\sqrt{\frac{1-j \cot \alpha}{2 \pi}} \Delta t e^{\frac{j}{2} \cot \alpha m^{2} \Delta u^{2}} \\
& \times \sum_{n=-N}^{N} y(n) e^{-j \csc \alpha n m \Delta u \Delta t} e^{\frac{j}{2} \cot \alpha n^{2} \Delta t^{2}}
\end{aligned}
$$

or using the transformation kernel $F_{\alpha}(m, n)$,

$$
Y_{\alpha}(m)=\sum_{n=-N}^{N} F_{\alpha}(m, n) y(n)
$$

The inverse transform is given for $M \geq N$ by

$$
y(n)=\sum_{m=-M}^{M} F_{\alpha}^{*}(m, n) Y_{\alpha}(m)
$$

Under the condition that

$$
\Delta t \Delta u=\frac{S 2 \pi \sin \alpha}{2 M+1}
$$

we obtain two definitions for the discrete FRFT for $\sin \alpha>0$ and $\sin \alpha<0$ cases:

$$
\begin{aligned}
Y_{\alpha}(m) & =\sqrt{\frac{\sin \alpha-j \cos \alpha}{2 M+1}} e^{\frac{j}{2} \cot \alpha m^{2} \Delta u^{2}} \\
& \times \sum_{n=-N}^{N} e^{-j \frac{2 \pi n m}{2 M+1}} e^{\frac{j}{2} \cot \alpha n^{2} \Delta t^{2}} y(n) \quad \sin \alpha>0
\end{aligned}
$$

and

$$
\begin{aligned}
Y_{\alpha}(m) & =\sqrt{\frac{-\sin \alpha+j \cos \alpha}{2 M+1}} e^{\frac{j}{2} \cot \alpha m^{2} \Delta u^{2}} \\
& \times \sum_{n=-N}^{N} e^{j \frac{2 \pi n m}{2 M+1}} e^{\frac{j}{2} \cot \alpha n^{2} \Delta t^{2}} y(n) \quad \sin \alpha<0
\end{aligned}
$$

For $M=N$ and $\alpha=\pi / 2$, the above definition reduces to DFT and for $\alpha=-\pi / 2$, it becomes the inverse DFT.

\section{CLOSED-FORM DISCRETE GABOR EXPANSION}

A fractional Gabor expansion with fraction order $\alpha$ can be defined for a discrete-time signal $x(n), n=0,1, \ldots N-1(N$ odd) as follows:

$$
x(n)=\sum_{m=0}^{M-1} \sum_{k=0}^{K-1} a_{m, k, \alpha} \tilde{h}_{m, k, \alpha}(n)
$$

where the fractional basis functions are

$$
\tilde{h}_{m, k}(n)=\tilde{h}(n-m L) K_{\alpha}(n, k)
$$

$M$ is the number of samples in time and $K$ is the number of samples in the $\alpha$ fractional domain (that is mixed time and frequency domain $u$ ); $L$ and $L^{\prime}$ are the samples in time and fractional $u$ domain respectively, and the previous condition still holds: $M L=K L^{\prime}=N$. Furthermore $K_{\alpha}(n, k)$ is the fractional kernel and it replaces the sinusoidal kernel $\left\{e^{j \omega_{k} n}\right\}$ of the traditional Gabor expansion where the term $e^{j \omega_{k} n}$ modulates synthesis window and shifts it in the frequency domain by $L$. Similarly, in the fractional case, the kernel $K_{\alpha}(n, k)$ will shift the window in the $u$ domain by the same step. A kernel which will provide such a shift can be obtained from the kernel of the closed-form discrete FRFT:

$$
\begin{aligned}
K_{\alpha}(n, k) & =\sqrt{\frac{\sin \alpha-j \cos \alpha}{N}} e^{\frac{j}{2}\left[n^{2} \Delta t^{2}+\left(k L^{\prime}\right)^{2} \Delta u^{2}\right] \cot \alpha} \\
& \times e^{-j \frac{2 \pi k L^{\prime}}{N} n}
\end{aligned}
$$

subject to the constraint that $\Delta t \Delta u=\frac{2 \pi|\sin \alpha|}{N}$. The fractional Gabor coefficients $a_{m, k, \alpha}$ will be calculated as before by an analysis window that is biorthogonal to $\tilde{h}(n)$. Then the Gabor coefficients are calculated as,

$$
a_{m, k, \alpha}=\sum_{n=-(N-1) / 2}^{(N-1) / 2} x(n) \tilde{\gamma}_{m, k, \alpha}^{*}(n)
$$

where the analysis basis functions $\tilde{\gamma}_{m, k, \alpha}(n)$ are obtained by

$$
\tilde{\gamma}_{m, k}(n)=\tilde{\gamma}(n-m L) K_{\alpha}(n, k)
$$

The completeness condition of this basis system is obtained by substituting Gabor coefficients $a_{m, k, \alpha}$ into the expansion in given (8):

$$
x(n)=\sum_{\ell=-(N-1) / 2}^{(N-1) / 2} x(\ell) \sum_{m=0}^{M-1} \sum_{k=0}^{K-1} \tilde{h}_{m, k, \alpha}(n) \tilde{\gamma}_{m, k, \alpha}^{*}(\ell)
$$


and the completeness condition is simplified to

$$
\begin{aligned}
\frac{1}{N} \sum_{m=0}^{M-1} \sum_{k=0}^{K-1} \tilde{h}(n-m L) & \tilde{\gamma}^{*}(\ell-m L) e^{\frac{j}{2}\left[n^{2}-\ell^{2}\right] \Delta t^{2} \cot \alpha} \\
& \times e^{-j \frac{2 \pi k L^{\prime}}{N}(n-\ell)}=\delta(n-\ell)
\end{aligned}
$$

The discrete fractional biorthogonality condition can be derived from the above completeness condition by Poisson sum formula as:

$$
\frac{1}{L L^{\prime}} \sum_{n=-(N-1) / 2}^{(N-1) / 2} \tilde{h}(n) \tilde{\gamma}^{*}(n+m K) e^{-j \frac{2 \pi k}{N} n}=\delta_{m} \delta_{k}
$$

for $0 \leq k \leq L-1,0 \leq m \leq L^{\prime}-1$ and $-(N-1) / 2 \leq n \leq$ $(N-1) / 2$. For a given Gauss synthesis window, the analysis window can be solved from the above equation system and used to calculate the fractional Gabor coefficients. Above fractional expansion is a generalization of the sinusoidal Gabor expansion, such that it reduces to the traditional Gabor for $\alpha=\pi / 2$

\section{ADAPTIVE SEARCH FOR THE OPTIMUM FRACTION ORDER}

Experiments show that, the time-frequency resolution performance of the fractional signal decomposition depends on the choice of $\alpha$. For a sinusoidal signal, the best representation may be obtained by $\alpha=\pi / 2$, whereas for a linear chirp, the appropriate choice of the fraction order depends on the chirprate. In the case of multi-component signals, the component that is matched by the analysis angle is better represented in the TF plane than the others. For an arbitrary signal, without apriori information on the frequency content, it is not possible to determine and select the appropriate fraction order $\alpha$. Therefore, in order to represent all components of a given multi-component signal with an acceptable TF resolution, we need a search algorithm for optimal analysis angles. This can be achieved by analyzing the signal with a set of pre-determined fraction orders $\left\{\alpha_{p}, 0 \leq p \leq P-1\right\}$. Then the Gabor coefficients can be chosen by maximizing a local energy concentration criteria $[4,9]$ in small time-frequency regions, such that only one component lies in. Hence an optimum fraction order is obtained by maximizing the following energy concentration measure in each of the $S \times Q$ blocks:

$E_{p}(m, k)=\frac{\sum_{s=-S / 2}^{S / 2-1} \sum_{q=-Q / 2}^{Q / 2-1} \Gamma_{p}^{2}(s-m L, q-k)\left|a_{m, k, \alpha_{p}}\right|^{4}}{\left(\sum_{s=-S / 2}^{S / 2-1} \sum_{q=-Q / 2}^{Q / 2-1} \Gamma_{p}^{2}(s-m L, q-k)\left|a_{m, k, \alpha_{p}}\right|^{2}\right)^{2}}$

for each of the Gabor coefficient sets $\left\{a_{m, k, \alpha_{p}}\right\}, p=$ $0,1, \cdots, P-1$. Then the Gabor coefficient subset that gives the maximum energy concentration $E_{p}(m, k)$ in each block is selected as the TF representation. Repeating this search procedure, whole TF plane is covered.

\section{EXAMPLES}

Example 1. In this example we analyze a signal composed of two crossing chirps. Fig. 1 shows the traditional magnitude squared Gabor coefficients $\left|a_{m, k}\right|^{2}$. Notice that both components are represented with a poor localization. Then we analyzed this signal using the proposed discrete fractional Gabor expansion with fraction orders $\alpha=[0-\pi]$, with $\pi / 20$ rad. increments. Then the resulting fractional Gabor coefficients are combined using the above local energy concentration measure, to obtain the final Gabor spectrum shown in Fig. 2. Notice that both components of the signal are represented in the TF plane with higher localization than the sinusoidal Gabor case.

Example 2. We have a signal composed of sinusoidal FM component and a linear chirp. Sinusoidal Gabor spectrum $\left|a_{m, k}\right|^{2}$ is given in Fig. 3. Notice that components of the signal cannot be represented properly in the TF domain. We also analyzed this signal using fraction orders in the interval $\alpha=[0-\pi]$ with $\pi / 36$ rad. increments. Then the adaptive search algorithm is used to find the fractional Gabor coefficients with the highest possible TF concentration in $40 \times 40$ TF blocks. The final Gabor spectrum is shown in Fig. 4.

Example 3. We consider another signal consisting of two quadratic FM components. Fig. 5 shows the combination of fractional Gabor coefficients obtained by the search algorithm.

\section{CONCLUSIONS}

In this paper, we present a method for fractional Gabor expansion for discrete-time, non-stationary signals. We give the completeness and biorthogonality conditions of this new expansion. Simulations show that the fractional method gives high resolution Gabor spectra if the analysis fraction order matches the frequency component of the signal. For an arbitrary signal, it is not possible to determine and select the appropriate fraction order $\alpha$. Therefore, in order to represent all components of a given multi-component signal with an acceptable TF resolution, we present a search algorithm for optimal analysis angles. The signal is analyzed with a set of fraction orders, and the Gabor coefficients are chosen for small TF blocks by maximizing a local energy concentration criteria yielding a highly localized final Gabor spectrum.

\section{REFERENCES}

[1] L. Cohen, Time-Frequency Analysis. Prentice Hall, Englewood Cliffs, NJ, 1995.

[2] F. Hlawatsch F. and G.F. Boudreaux-Bartels, "Linear and quadratic time-frequency signal representations," IEEE Signal Proc. Magazine, Vol. 9, No. 2, pp. 21-67, Apr. 1992.

[3] J. Wexler, and S. Raz, "Discrete Gabor Expansions," Signal Processing, Vol. 21, No. 3, pp. 207-220, Nov. 1990.

[4] D.L. Jones, and T.W. Parks, "A High Resolution Data-Adaptive Time-Frequency Representation," IEEE Trans. on Signal Proc., Vol. 38, No. 12, pp. 2127-2135, Dec. 1990.

[5] S. Mallat, and, Z. Zhang, "Matching Pursuit with TimeFrequency Dictionaries," IEEE Trans. on Signal Proc., Vol. 41, pp. 3397-3415, Dec. 1993.

[6] R.G. Baraniuk, and D.L. Jones, "Shear Madness: New Orthonormal Bases and Frames Using Chirp Functions," IEEE Trans. on Signal Proc., Vol. 41, No. 12, pp. 3543-3549, Dec. 1993. 
[7] A. Akan, and L.F. Chaparro, "Multi-window Gabor Expansion for Evolutionary Spectral Analysis," Signal Processing, Vol. 63, pp. 249-262, Dec. 1997.

[8] A. Bultan, "A Four-Parameter Atomic Decomposition of Chirplets," IEEE Tans. on Signal Proc., Vol. 47 pp. 731-745, 1999.

[9] A. Akan, and L.F. Chaparro, "Evolutionary Chirp Representation of Non-stationary Signals via Gabor Transform,", Signal Processing, Vol. 81, No. 11, pp. 24292436, Nov. 2001.

[10] A.J. van Leest, and M.J. Bastiaans, “Gabor's Signal Expansion and the Gabor Transform on a Non-separable Time-Frequency Lattice," J. Franklin Institute, Special Issue on Time-Frequency Signal Analysis and its Applications, Vol. 337, No. 4, pp. 291-301, Jul. 2000.

[11] S.C. Pei, and J.J. Ding, "Closed-Form Discrete Fractional and Affine Fourier Transform," IEEE Trans. on Signal Proc., Vol. 48 pp. 1338-1353, 2000.

[12] A. Akan, and Y. Cekic, "A Fractional Gabor Expansion," Journal of The Franklin Institute, Vol. 340, No. 5, pp. 391-397, Nov. 2003.

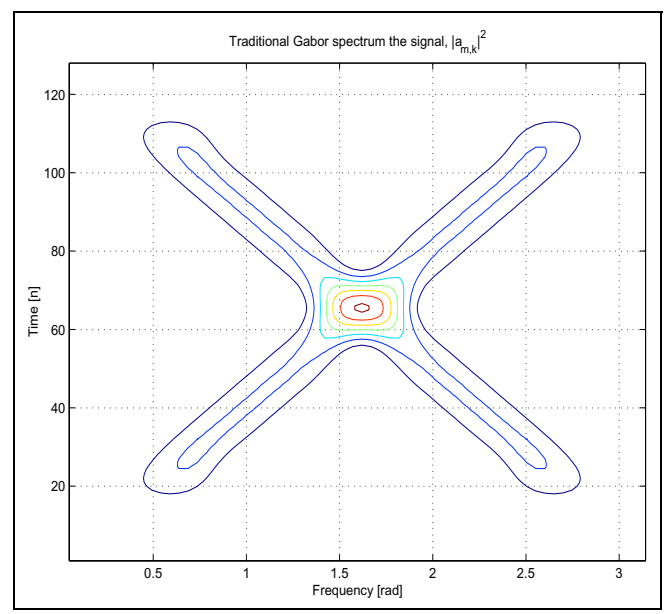

Figure 1: Sinusoidal Gabor spectrum of the signal

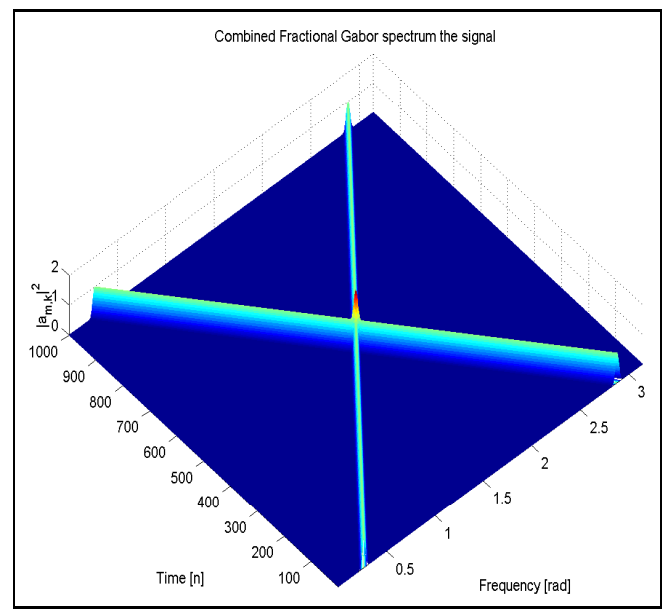

Figure 2: Combined Fractional Gabor spectra

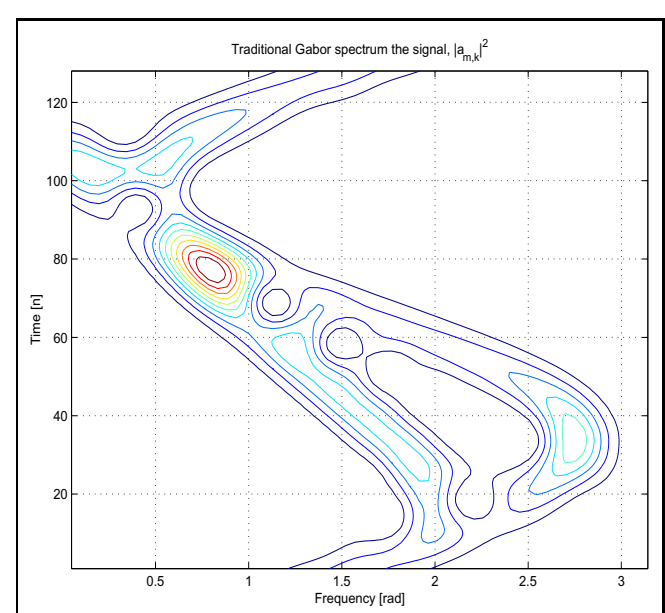

Figure 3: Traditional Gabor spectrum of a sinusoidal FM and a chirp

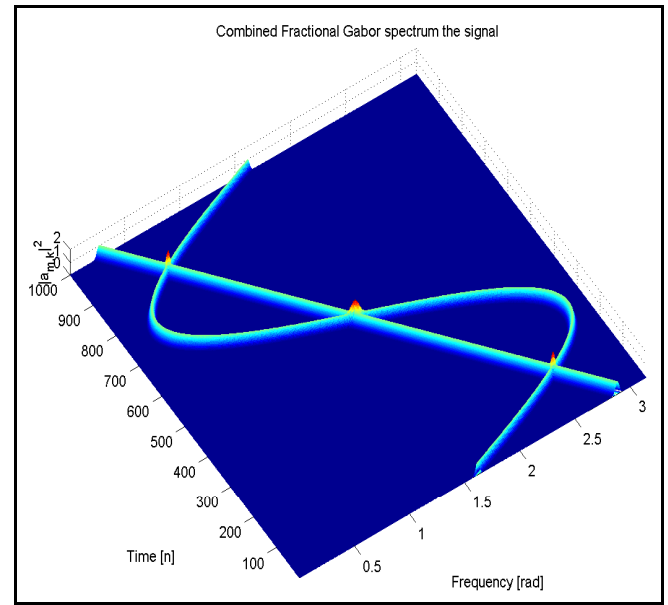

Figure 4: Combination of Fractional Gabor spectra

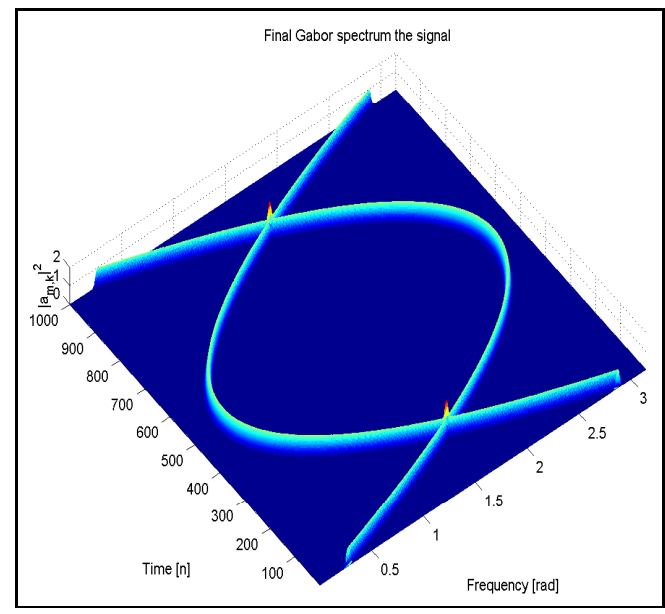

Figure 5: Two quadratic FM signals 